\title{
Les deux premières années des nouvelles institutions écossaises
}

The First Two Years of the New Scottish Institutions

Edwige Camp

\section{(2) OpenEdition}

1 Journals

Édition électronique

URL : http://journals.openedition.org/rfcb/698

DOI : $10.4000 /$ rfcb.698

ISSN : 2429-4373

Éditeur

CRECIB - Centre de recherche et d'études en civilisation britannique

\section{Édition imprimée}

Date de publication : 1 février 2002

Pagination : $31-43$

ISBN : 2-911580-13-3

ISSN : 0248-9015

Référence électronique

Edwige Camp, "Les deux premières années des nouvelles institutions écossaises ", Revue Française de Civilisation Britannique [En ligne], XI-3 | 2002, mis en ligne le 21 mars 2016, consulté le 20 avril 2019. URL : http://journals.openedition.org/rfcb/698 ; DOI : 10.4000/rfcb.698

Ce document a été généré automatiquement le 20 avril 2019.

\section{(c) $(1)$}

Revue française de civilisation britannique est mis à disposition selon les termes de la licence Creative Commons Attribution - Pas d'Utilisation Commerciale - Pas de Modification 4.0 International. 


\title{
Les deux premières années des nouvelles institutions écossaises
}

\author{
The First Two Years of the New Scottish Institutions
}

Edwige Camp

1 La réforme constitutionnelle entrée en vigueur le 6 mai 1999 avec l'élection du Parlement écossais, prévue par le Scotland Act 1998 ${ }^{1}$, et qualifiée de devolution, est définie comme un transfert de pouvoir d'une autorité supérieure à une autorité de rang inférieur. Elle ne permet pas de retrouver la situation antérieure à 1707, avec deux Parlements distincts et un même souverain, car elle instaure un Parlement écossais constitutionnellement subordonné à celui de Westminster issu de l'Union². L'Écosse, dont les affaires étaient jusqu'en 1999 gérées par un ministère (The Scottish Office) créé en 1885 et dont les pouvoirs ont été progressivement étendus, dispose désormais d'institutions politiques propres. La réforme consacre la place prééminente du Parlement, affaiblissant par contrecoup la position centrale qu'occupaient traditionnellement le système judiciaire et l'Église presbytérienne, garants de l'identité écossaise dans le traité d'Union. Toutefois, ce Parlement, conçu au sein d'une Convention constitutionnelle entre partis d'opposition au gouvernement central conservateur (le SNP s'en est retiré), apparaît comme un moyen de remédier aux dysfonctionnements du Parlement de Westminster et de prendre en compte le contexte écossais. Ainsi, trois aspects essentiels caractérisent les nouvelles institutions écossaises : le rôle central du Parlement, les relations avec les institutions britanniques, et la coalition entre les travaillistes et les libéraux-démocrates.

\section{Le rôle central du Parlement}

2 Les Écossais se sont dotés d'un Parlement monocaméral, dans lequel siègent leurs quatre principaux partis ( 55 travaillistes, 35 nationalistes, 19 conservateurs et 17 libérauxdémocrates) auxquels s'ajoutent trois « indépendants » représentant les Verts, le Scottish Socialist Party et un ancien député travailliste qui n'avait pas obtenu sa ré-investiture lors des élections de Westminster ${ }^{3}$. La présence de ceux-ci, ainsi que la quasi-proportionnalité entre suffrages et sièges obtenus par les opposants aux travaillistes (les écarts étant 
inférieurs à deux points) et surtout l'absence de majorité absolue, sont dus au mode de scrutin qui associe le système majoritaire à un tour dans 73 circonscriptions inchangées par rapport à celles du Parlement de Westminster (si l'on excepte Orkney and Shetland, qui était scindée en deux), et l'élection de 56 députés supplémentaires à la proportionnelle, sur des listes régionales. Or, tous ces députés ont les mêmes droits. Néanmoins, sur le terrain, les élus directs sont à l'origine plus facilement sollicités par les électeurs pour résoudre leurs problèmes concrets, en raison de leur mode de désignation. En outre, ces députés, pour la plupart travaillistes, coopèrent difficilement avec leurs collègues des autres partis issus du scrutin de liste, lesquels recherchent une notoriété qui leur permettrait, lors de prochaines élections, de se présenter directement aux suffrages des électeurs ${ }^{4}$.

3 Le Parlement écossais est organisé selon des modalités définies par les partis, sur la base du Scotland Act 1998, au sein d'un Consultative Steering Group, dont le rapport proposait une Chambre dotée de pouvoirs effectifs, et ouverte sur la société civile. D'une part, s'agissant des premiers, en l'absence d'une deuxième Chambre, une grande place est accordée aux commissions. En effet, elles sont permanentes et cumulent les fonctions de vérification du travail gouvernemental et d'examen des projets et propositions de loi avant leur discussion en session plénière ; elles ont également des pouvoirs d'initiative législative et d'enquête. Il existe deux catégories de commissions, par thème d'action politique et par fonction parlementaire ${ }^{5}$. En outre, afin d'éviter la domination du parti majoritaire, et plus généralement de l'esprit partisan, leurs membres sont élus à la proportionnelle des groupes (intégrant les trois indépendants), et leurs présidents ne peuvent agir contre la volonté de leurs collègues. De même, certaines commissions (comme celle des Finances) ont instauré des modalités de composition par ordre alphabétique et non par parti, et certains partis, tels les libéraux-démocrates, évitent que leurs porte-parole ne président des commissions ${ }^{6}$. Une préoccupation semblable est à l'origine du choix d'un hémicycle, censé faciliter la recherche du consensus ${ }^{7}$.

Fonction centrale du Parlement, le contrôle de l'exécutif s'exerce dès la constitution du gouvernement, laquelle doit obtenir l'aval du Parlement. Néanmoins, le premier ministre répartit librement les postes ministériels et décide de leur durée. Seuls les deux conseillers juridiques (Lord Advocate et Solicitor General for Scotland), qui font nécessairement partie de l'exécutif, peuvent ne pas être députés, mais leur nomination, et leur renvoi, doivent être approuvés par le Parlement. Ce dernier participe également à la nomination d'autorités administratives et judiciaires susceptibles de remettre en cause l'activité de l'exécutif ${ }^{8}$. Ensuite, en cours de législature, les députés débattent de divers sujets, éventuellement à l'issue d'une déclaration de l'exécutif, et posent de nombreuses questions susceptibles de contraindre ce dernier à s'expliquer, par exemple s'agissant du coût et de l'avancement de la construction du nouveau bâtiment du Parlement ${ }^{9}$. De plus, les ministres écossais sont fréquemment auditionnés par les commissions. Jack McConnell, ministre des Finances, a ainsi assisté la première année à huit des vingt-neuf réunions de la commission chargée des questions financières. Ces sollicitations sont parfois mal acceptées par l'exécutif, mais seuls les deux conseillers juridiques peuvent les refuser ${ }^{10}$. Or le vote des commissions a pu contraindre l'exécutif à modifier ses projets, pour accepter un amendement (les questions sur la religion dans le prochain recensement), ou un texte (la proposition, présentée par le député SSP Tommy Sheridan, d'abolition des ventes aux enchères de biens saisis pour recouvrer les dettes). En cas d'échec, les commissions pourront déposer leur propre proposition de loi, voire, 
éventuellement, une motion de défiance (tous les membres de l'exécutif étant alors solidairement responsables). Enfin, le Parlement est élu pour quatre ans, et seul son Président, et non l'exécutif, peut proposer sa dissolution anticipée à la demande des deux-tiers des députés ou en cas de vacance du poste de premier ministre supérieure à 28 jours, ce qui a obligé les travaillistes, pris au dépourvu par le décès de Donald Dewar en octobre 2000, à écourter la campagne interne permettant de désigner son successeur Henry McLeish. En effet, si ce dernier, ancien président de la CRG, s'était largement impliqué lors de l'élaboration du Scotland Act 1998, il ne s'imposait pas autant que son prédécesseur, qui, pendant plus de vingt ans, avait travaillé à l'aboutissement de cette réforme. Il convient de souligner le rôle prééminent du Président dans les désignations, les vérifications et l'organisation du travail parlementaire ${ }^{11}$. La fonction est exercée par le libéral-démocrate David Steel, peu contesté en raison de sa longue expérience de député et de militant en faveur de la réforme constitutionnelle.

D'autre part, le Parlement écossais se caractérise par sa volonté d'ouverture sur la société civile. Tout d'abord, les commissions se réunissent (parfois) et enquêtent (plus fréquemment) dans toute l'Écosse. Ensuite, le Parlement reçoit de nombreuses pétitions, traitées par une commission fonctionnelle spécialisée qui les réorientera éventuellement vers la commission thématique compétente ${ }^{12}$. Enfin, dès la publication d'un Livre blanc, l'avis des personnes intéressées est sollicité et publié dans la note accompagnant le projet afin d'expliquer celui-ci en termes compréhensibles. Ce procédé a incité l'exécutif à renoncer aux péages sur l'autoroute reliant Glasgow et Edimbourg, mode de financement d'ailleurs très controversé qui explique le délai de dix-huit mois entre le dépôt du texte et son adoption (récente). En outre, le Parlement devrait également contribuer à l'application de la loi sur la liberté d'information, par la désignation du commissaire. Par ailleurs, les groupes de pression peuvent obtenir le dépôt d'une proposition de loi, voire mener leur propre campagne pour obtenir le retrait d'un texte. Mais les moyens considérables d'opposition aux textes visant respectivement à l'abolition de l'article 28/2A de la loi sur les collectivités locales de 1986, interdisant l'évocation de l'homosexualité dans les organismes dépendant de celles-ci, notamment les écoles, et à l'interdiction de la chasse à courre, ont échoué ${ }^{13}$.

\section{Les relations avec les institutions britanniques}

Le Parlement d'Écosse étant créé par une loi votée par le Parlement de Westminster, il est subordonné à celui-ci ; certes, contrairement au projet de la fin des années soixante-dix, il n'est plus qualifié de simple Assemblée, ses membres sont des Members of Parliament, (seul un 'S' pour Scottish étant ajouté) ${ }^{14}$, et, ses pouvoirs n'étant pas précisés (il s'agit en fait de ceux conférés au Scottish Office jusque-là), ils ne semblent pas limités. Toutefois, il ne dispose pas des compétences régaliennes, ne peut voter ni sur les matières réservées au Parlement britannique ${ }^{15}$ (mais il en discute, notamment des affaires européennes), ni dans le domaine constitutionnel ${ }^{16}$. À l'inverse, le Parlement de Westminster peut intervenir sur toute question, sans restriction, même si, par convention (dite Sewell), il consultera au préalable son homologue écossais s'agissant de domaines relevant de ce dernier (comme la création d'une Agence de sécurité alimentaire), ou de questions réservées influant sur des matières "dévolues" (telle la réforme de la gestion des demandes d'asile $)^{17}$. Enfin, au cours du processus législatif, la protection des domaines réservés est assurée par l'examen des textes écossais, à trois étapes, par des autorités 
politiques, juridiques, voire judiciaires ${ }^{18}$. À ce jour, aucun conflit de compétences n'a été soulevé ${ }^{19}$. Cependant, ces divers contrôles doivent permettre aux décisions écossaises de s'appliquer, selon l'interprétation des textes la plus restrictive possible.

De même, l'exécutif écossais dispose de pouvoirs réglementaires dans les domaines dévolus au Parlement ${ }^{20}$. Toutefois, l'exécutif dispose d'un statut moindre que celui du Parlement ainsi que l'implique son titre même (il s'agit d'un exécutif et non d'un gouvernement, avec à sa tête un First et non un Prime Minister) ${ }^{21}$. Son autonomie est, en outre, limitée par le pouvoir conféré au secrétaire d'État pour l'Écosse d'interdire ou de modifier directement tout acte réglementaire. Afin de prévenir, voire de régler, les différends éventuels avec le gouvernement britannique, ce dernier a élaboré des mécanismes ${ }^{22}$ s'ajoutant à la coopération régulière, et peut modifier la répartition des compétences. Par ailleurs, il demeure seul responsable de la politique communautaire, même s'il collabore avec l'exécutif écossais qu'il autorise parfois à représenter le Royaume-Uni dans des domaines importants pour l'Écosse, comme la pêche ${ }^{23}$. En outre, les ministres britanniques ne sont tenus de s'expliquer devant les commissions écossaises que sur des matières "dévolues " mais partagées, pour leur éviter d'avoir à rendre compte devant deux Parlements. Ainsi, le Chancelier de l'Échiquier Gordon Brown et le Secrétaire d'État pour l'Écosse John Reid ont refusé de s'exprimer sur l'utilisation des fonds structurels européens ${ }^{24}$.

Le financement des institutions écossaises est également assuré par les institutions britanniques, en partie à l'aide de la formule Barnett ${ }^{25}$, utilisée jusque-là pour le Scottish office. Cette formule concerne uniquement les matières conférées au Parlement écossais et les augmentations du budget (dont le niveau initial n'est pas remis en cause), qui seront alignées sur celles des ministères anglais correspondants ${ }^{26}$, même si la répartition entre secteurs est effectuée par les Écossais. Cette formule ne figure pas dans le Scotland Act 1998, mais le gouvernement continue à la défendre et à l'utiliser, et le programme triennal de dépenses annoncé par G. Brown au cours de l'été 2000 ne fait pas exception, même s'il confirme la réalisation progressive de l'un des objectifs initiaux, à savoir, la réduction relative du budget écossais ${ }^{27}$. L'avantage financier dont bénéficie ainsi l'Écosse, qui peut atteindre $20 \%$ pour certaines dépenses identifiables dépendant de la formule (la santé, à la différence de la Sécurité sociale), s'explique par ses besoins spécifiques (faible densité de la population, santé relativement mauvaise, économie concurrentielle mais souvent affaiblie par sa dépendance passée à l'égard du secteur secondaire et sa dépendance actuelle envers les entreprises étrangères propriétaires des multiples sociétés de services). Cet avantage financier est parfois critiqué en Angleterre, surtout au Nord-Est, mais n'est pas réellement remis en cause, d'autant que l'esprit des réformes semble empêcher toute régression des dépenses publiques écossaises. Qui plus est, les nationalistes estiment que c'est l'Écosse qui subventionne le reste du Royaume-Uni grâce au pétrole de la mer du Nord, et leurs pressions, liées à leur poids politique, ont une influence non négligeable sur le budget écossais et sur le débat, désormais centré sur une éventuelle autonomie fiscale. En outre, le Parlement écossais ne peut faire varier l'impôt que d'une façon limitée ${ }^{28}$, l'exécutif écossais s'étant, par ailleurs, engagé à ne pas recourir à cette possibilité pendant la première législature, alors qu'il ne dispose pas d'autres ressources propres ${ }^{29}$, tandis qu'il doit régulièrement rendre des $\operatorname{comptes}^{30}$. Néanmoins, en dépit de ces contraintes, les institutions écossaises ont effectué des choix singuliers, comme l'abolition des droits d'inscription universitaire, et ont conçu un dispositif original permettant le remboursement des droits universitaires à l'issue des études, en 
fonction des revenus des diplômés, les sommes ainsi perçues servant à financer des bourses pour les étudiants les plus défavorisés. Cependant, les fonds initiaux proviennent du budget du ministère écossais concerné, et non d'un transfert, alors qu'il s'agissait d'une mesure populaire, ce qui suscite certaines interrogations quant au sort de politiques plus controversées, telles la gratuité des frais d'assistance personnelle aux personnes âgées en centre de long séjour et la forte augmentation des salaires des enseignants, annoncées en début d'année par H. McLeish, sur la base des rapports Sutherland et McCrone ${ }^{31}$.

9 L'Écosse a conservé sa représentation au sein des institutions britanniques, le gouvernement souhaitant éviter toute rupture dans un premier temps. Seule est prévue une réduction du nombre des députés écossais au Parlement de Westminster, qui passerait de 72 à une soixantaine, ce qui refléterait plus fidèlement le poids relatif de l'Écosse dans la population du Royaume, mais entraînerait du même coup la diminution $\mathrm{du}$ nombre des $\mathrm{MSP}^{32}$. Il reste que le rôle des députés écossais siégeant à Westminster dans les affaires du pays se trouve affaibli. Leurs électeurs auront tendance à s'adresser aux MSP de leur circonscription, les MP écossais n'intervenant plus dans les questions qui sont au cœur des préoccupations quotidiennes des Écossais même s'ils font souvent de leur mieux pour être à l'écoute de leurs problèmes. Au Parlement de Westminster, leur rôle tend à se limiter au contrôle du Scottish Office (de taille réduite) et des ministres britanniques dans l'exercice des pouvoirs réservés, et à la participation aux débats et enquêtes portant sur ces mêmes domaines, dans le but d'attirer l'attention des médias écossais désormais plutôt centrée sur le Parlement d'Édimbourg ${ }^{33}$. De plus, les 15 MP élus au Parlement écossais en 1999 ont marqué une nette préférence pour leur second mandat au point de renoncer, à l'exception d'Alex Salmond, ancien leader du SNP ${ }^{34}$, à se présenter aux élections de juin 2001. D'autres MP écossais ont choisi de se spécialiser dans le domaine des affaires étrangères ou britanniques. Or, leur droit de vote dans des domaines qui relèvent en Écosse des institutions écossaises (question dite de West Lothian) n'est plus réellement remis en cause ${ }^{35}$. Il est vrai qu'en votant le budget de ministères anglais, les MP écossais contribuent en fait à la détermination d'une (faible) partie du budget écossais, tandis que la majorité travailliste est telle qu'aucun vote n'a été acquis grâce aux seules voix écossaises. Qui plus est, en pratique, les Écossais ont obtenu des postes éminents dans les domaines réservés, comme Gordon Brown ou Robin Cook (Affaires étrangères) ${ }^{36}$ ou non partisans (Michael Martin devenant Speaker). En outre, les MP ne sont plus censés poser de questions relatives aux matières gérées en Écosse, restriction toutefois appliquée de façon très souple.

Quant au secrétaire d'État, il conserve la représentation des intérêts écossais à Londres, celle des intérêts britanniques en Écosse, et il assure la coopération entre les deux gouvernements (entre lesquels les cumuls de fonctions sont interdits, à la différence du régime applicable aux parlementaires). De plus, J. Reid, et Helen Liddell qui lui a succédé au début de 2001, ont refusé de devenir des ministres secondaires: après avoir fait modifier le nom du Scottish Office (devenu Scotland Office), et obtenu un doublement du budget initialement alloué au ministère, ils sont fréquemment intervenus pour préserver les emplois écossais ${ }^{37}$, ou bien l'unité du Royaume-Uni ${ }^{38}$. Il s'agit aussi pour ces fortes personnalités de démontrer leur utilité, car le rang, voire la nature du secrétariat d'État pour l'Écosse, suscitent certaines controverses ${ }^{39}$. Cet activisme a engendré des tensions avec l'exécutif écossais, accentuées par les médias écossais qui ne cessent d'évoquer l'existence de «guerres de territoires » depuis la demande d'enquête parlementaire de 
D. Dewar face aux accusations portées contre le fils de J. Reid ${ }^{40}$. Plus généralement, les ministres britanniques, et notamment le premier d'entre eux, cherchent à promouvoir un esprit de coopération, toute tentative d'ingérence étant exploitée par le SNP au bénéfice de la cause indépendantiste ${ }^{41}$. Seul le coût élevé de certaines mesures écossaises est régulièrement souligné. De plus, des ministres non écossais initialement hostiles à la réforme travaillent avec leurs homologues, même libéraux-démocrates ${ }^{42}$.

Or les nouvelles institutions doivent également s'affirmer, notamment en défendant les intérêts économiques de l'Écosse, en reléguant, le cas échéant, au second plan le rôle du gouvernement britannique ${ }^{43}$, ou encore en faisant connaître la position écossaise dans les domaines réservés, comme la monnaie européenne ${ }^{44}$. Plus généralement, l'examen des dix-neuf lois adoptées jusqu'en juin 2001 fait apparaître un souci de traiter de questions spécifiquement écossaises, comme l'abolition des droits féodaux sur la terre, l'interdiction des ventes aux enchères, le mode de nomination des juges ${ }^{45}$, ou d'apporter des solutions écossaises à des problèmes plus généraux, comme le financement des études universitaires, la suppression de l'article 28, ou la liberté d'information ${ }^{46}$. En 2001, les derniers engagements financiers du premier ministre, suivis d'un projet facilitant l'achat par les crofters de leurs terres et d'un refus d'étendre l'implication du secteur privé dans le service public de santé en Écosse comme le propose Tony Blair, s'inscrivent dans ce mouvement, même si certains choix relèvent aussi de préoccupations plus populistes que celles de son prédécesseur, Donald Dewar, souvent jugé trop prudent. De plus, trois lois sont issues de propositions (outre celle de T. Sheridan, elles concernent la pêche et les baux emphytéotiques), et, en juin 2001, une première proposition a été déposée par une commission (concernant la violence conjugale), démontrant la volonté d'action des MSP de tous partis face à l'exécutif. Par ailleurs, certaines solutions écossaises alimentent le débat national et peuvent même influer sur les décisions des dirigeants britanniques ${ }^{47}$.

\section{La coalition entre les travaillistes et les libéraux- démocrates}

Une autre originalité des nouvelles institutions écossaises est leur gestion par une coalition entre les travaillistes et les libéraux-démocrates. Cette coalition était inéluctable en raison du mode de scrutin adopté par la Convention constitutionnelle, qui avait de surcroît permis à D. Dewar de nouer des relations de confiance avec J. Wallace, le leader des libéraux-démocrates écossais. Ainsi, le 14 mai 1999, une semaine seulement après les élections, les deux hommes publiaient un accord de partenariat, résultant de négociations dont l'issue était quasiment certaine, car, entre-temps, les nominations de D. Dewar et D. Steel avaient été votées par les députés des deux partis (malgré l'importance que les travaillistes attachent à la fonction de président), tandis que les deux leaders des partis britanniques apportaient leur soutien, sans intervenir directement ${ }^{48}$. Deux éléments controversés seront traités, car ils sont jugés essentiels par les libéraux-démocrates: l'utilisation des pouvoirs fiscaux du Parlement (écartée), et l'abolition des droits d'inscription des étudiants (finalement acceptée) ${ }^{49}$. Qui plus est, les libéraux-démocrates ne se contentent pas de soutenir les travaillistes au Parlement, ils obtiennent des postes ministériels avantageu ${ }^{50}$. Wallace est ainsi nommé vice-premier ministre et ministre de la Justice, symbole de la participation effective de sa formation, et moyen de prévention des conflits. Cette décision évite également à $\mathrm{D}$. Dewar la désignation d'un successeur au sein de son parti, poste convoité, notamment par H. McLeish. Or, Wallace aura l'occasion 
de faire fonction de premier ministre, lors de l'hospitalisation (en mai 2000) puis à la suite du décès de $\mathrm{D}$. Dewar, et il aura su s'imposer dès la première séance de questions orales, malgré les interrogations de certains travaillistes sur sa légitimité politique ${ }^{51}$. En outre, D. Dewar a procédé à la répartition des portefeuilles ministériels, utilisant les ministres délégués pour assurer un équilibre politique, et son successeur fera de même ${ }^{52}$. Toutefois, l'exécutif est peu expérimenté, ce qui n'est pas seulement imputable aux libérauxdémocrates, ni aux choix initiaux. En effet, seuls quatre ministres avaient exercé des fonctions semblables ${ }^{53}$, et certains, occupant parfois des postes essentiels, sont très jeunes ${ }^{54}$. Cette relative inexpérience a posé quelques problèmes de communication à l'exécutif, face à des médias écossais très critiques à l'égard d'institutions proches. Ainsi, D. Dewar, à l'origine partisan d'un contrôle strict sur son Cabinet, et d'une limitation du nombre des conseillers spéciaux, a finalement cédé aux pressions des jeunes travaillistes réputés ambitieux (tel H. McLeish), qui cherchent à promouvoir leur action par des moyens propres $^{55}$.

13 La coalition a également permis aux libéraux-démocrates d'obtenir des concessions libérales et sociales de la part des travaillistes ${ }^{56}$, qui ont facilité la tâche du premier ministre dans le dossier des personnes âgées ${ }^{57}$. Toutefois, le groupe parlementaire libéraldémocrate est moins discipliné que le groupe travailliste ${ }^{58}$. Ainsi, sur un sujet jugé important, la pêche, les libéraux-démocrates ont multiplié les demandes d'explication à leur ministre et ont contribué, en mars 2001, à la première adoption d'un amendement contre la volonté de l'exécutif ${ }^{59}$, défaite certes provisoire, mais qui a incité $\mathrm{H}$. McLeish à rappeler la discipline indispensable au partenariat, d'autant que treize travaillistes étaient absents. Cependant, ceux-ci évitent tout vote susceptible de constituer une réelle menace pour leur parti ${ }^{60}$.

14 Ainsi, les Écossais ont créé des institutions influencées par le modèle britannique, mais autonomes, malgré les critiques virulentes, sans doute proportionnelles aux attentes. La campagne électorale britannique de juin 2001 a confirmé la force des liens entre l'Écosse et le reste du Royaume-Uni ${ }^{61}$, tout en mettant en évidence le caractère irréversible du transfert de pouvoir. En effet, tous les partis sont désormais favorables aux institutions écossaises, les travaillistes et les libéraux-démocrates parvenant à dissocier leurs relations selon les situations locales, sans être pénalisés par les électeurs, contrairement à ce que semblaient indiquer les élections partielles ${ }^{62}$. Les nationalistes, qui constituent la principale force d'opposition, cherchent à utiliser les institutions, où ils ont su se faire apprécier, pour négocier le passage à l'indépendance ${ }^{63}$. Quant aux conservateurs, initialement opposés à toute réforme, ils présentent des propositions constitutionnelles et socio-économiques concrètes, d'autant qu'ils doivent leur représentation aux nouvelles institutions ${ }^{64}$. Ainsi, la devolution s'affirme effectivement comme un processus, et pas seulement comme un événement ponctuel ${ }^{65}$ et figé (les institutions ont déjà connu des adaptations), résultant de l'action de leaders historiques (notamment D. Dewar) ou de décisions prises à Londres. 


\section{NOTES}

1. Scotland Act, chap. 46, 1998. Les commentaires et illustrations du texte sont fondés sur les analyses de C. HIMSWORTH \& C. MUNRO, The 1998 Scotland Act, Édimbourg: W. Green, 2000, $2^{\mathrm{e}}$ édition, 302 p., et de A. PAGE, C. REID \& A. ROSS, A Guide to the Scotland Act 1998, Édimbourg: Butterworths, 1999, $326 \mathrm{p}$.

2. cf. V.BOGDANOR, Devolution in the United Kingdom, Oxford : Oxford University Press, 1999, pp. 15 et 283. Cette analyse s'oppose à ce qu'a affirmé Winnifred Ewing, élue britannique et européenne de longue date du Scottish National Party (SNP), chargée d'ouvrir la première session du Parlement en sa qualité de doyenne.

3. Dennis Canavan représentait le parti à la Chambre des Communes depuis 1974. Nos chiffres tiennent compte des résultats des élections partielles qui ont eu lieu depuis mai 1999.

4. Or les règles posées par George Reid, vice-président du Parlement, interdisent seulement l'empiètement manifeste. Les travaillistes ont souhaité réduire de moitié les allocations des députés de liste, mais ils ont accepté un partage limité à leurs bureaux. P. LYNCH, Scottish Government and Politics, Édimbourg : Edinburgh University Press, 2001, pp. 55-56.

5. Au début de 2001, elles sont passées de 8 à 9 dans chaque catégorie, et ont subi quelques modifications, en raison d'une surcharge de travail (la Commission de la Justice étant scindée en deux), de l'importance croissante d'un secteur (avec la création d'une Commission des private bills ), ou de la nécessité d'élargir les attributions (les affaires rurales devenant par exemple le développement rural, tandis que la justice sociale remplace la lutte contre la pauvreté, le logement et le secteur associatif). En effet, le travail des commissions est très inégal : la première année, la Commission de la Justice a reçu un grand nombre de projets de loi, des pétitions, et a disposé de ce fait d'un temps moindre à consacrer aux enquêtes qui ont constitué l'essentiel de l'activité des commissions chargées du transport, de l'environnement, des entreprises et de la formation, auxquelles aucun projet de loi n'a été transmis pour examen. De plus, elle comptait en son sein de nombreux juristes, dont la présidente, Roseanna Cunningham, par ailleurs porteparole éminente du SNP. Ibid., p. 75.

6. Les conservateurs et surtout les nationalistes conservent des porte-parole virulents comme présidents de commissions, mais le travail est plus serein depuis le retrait de John Swinney, devenu leader du SNP en septembre 2000.

7. cf. David STEEL “A Dozen Differences of Devolution”, discours prononcé à l'Union Society d'Oxford, 4/6/01.

8. Il doit nommer le commissaire chargé de faire respecter le droit d'accès aux informations détenues par des autorités publiques, conformément au texte sur la liberté d'information actuellement en discussion. Il intervient dans les décisions mettant fin aux fonctions des juges des principaux tribunaux, après avis d'une commission indépendante, résultant d'un amendement au projet initial du Scotland Act 1998 destiné à renforcer la protection d'un pouvoir judiciaire appelé à intervenir plus fréquemment dans l'activité politique.

9. Donald Gorrie (LD) et Margo McDonald (SNP) ont été particulièrement tenaces. 8500 questions ont été posées la première année selon G. LEICESTER “Scotland" in R.HAZELL (ed.), The State and the Nations, Thorverton: Imprint Academic, p. 22 ; les questions orales sont plus fréquemment spontanées (et contraignantes) depuis l'adoption de nouvelles règles fin 1999. De plus, les MSP exigent d'être les premiers destinataires d'une déclaration ministérielle ; ainsi, au début de 2001, Angus MacKay (Finances) n'a pu effectuer la sienne car il en avait au préalable communiqué le 
texte aux médias, cf. E. BORT "The Scottish Parliament in a Run-up to a General Election”, Scottish Affairs, $\mathrm{n}^{\circ} 35$, printemps 2001, p. 35.

10. Ils invoqueront une enquête en cours ou l'intérêt public (article 29). D. Dewar a critiqué les nombreuses comparutions de deux ministres, Susan Deacon et Rhona Brankin. Voir R. PYPER "The First Minister and the Scottish Executive" in G. HASSAN \& C.WARHURST (eds.), The New Scottish Politics, Édimbourg : The Stationery Office, 2000, p. 80.

11. Outre les pouvoirs qu'il détient s'agissant de la recommandation du premier ministre, de la désignation d'un faisant fonction en cas de vacance provisoire et de dissolution du Parlement, le Président, qui est le représentant ès qualités du Parlement, veille au respect des domaines de compétence du Parlement (voir infra), et à la bonne organisation du travail parlementaire. Il est assisté de deux vice-présidents.

12. Sur les 381 pétitions reçues jusqu'en juin 2001, la proportion de transmission est passée de la moitié la première année à un cinquième; ces pétitions dénoncent régulièrement des dysfonctionnements des quangos dans le secteur de la santé. L'une d'entre elles, défendant les services d'urgence de l'hôpital de Stobhill menacés de fermeture, a été à l'origine, lors de l'élection partielle de juin 2001 destinée à remplacer Sam Galbraith démissionnaire du Parlement écossais pour raisons de santé, de la candidature d'un médecin qui a obtenu près d'un cinquième des suffrages (The Herald, 9/6/01).

13. Ainsi, Brian Soutar, chef d'entreprise, a organisé un référendum, mais l'exécutif a maintenu la suppression de l'article $28 / 2 \mathrm{~A}$, tout en conseillant d'évoquer la valeur particulière du mariage. De même, la Countryside Alliance a essayé, sans succès, d'obtenir le retrait de la proposition de Mike Watson visant l'interdiction de la chasse à courre, par des moyens judiciaires (pour abus de pouvoir par rapport au Scotland Act 1998) et parlementaires (pour manquement à la déontologie de son promoteur, qui, faute de moyens, avait dû solliciter une aide extérieure pour rédiger le texte de la proposition), cf. M. WATSON, Year Zero, Édimbourg : Polygon, 2001, pp. 51-52 et 176. Ce second texte n'est toutefois pas encore adopté, contrairement au premier.

14. Ils prêtent également serment à la Couronne à leur entrée en fonction (ce qui a donné lieu à quelques écarts gestuels de T. Sheridan) mais, à la différence des MP, ils peuvent être élus parmi les pairs, ce qui est le cas de M. Watson, D. Steel ou James Douglas-Hamilton, d'ailleurs anciens MP. B. TAYLOR, The Scottish Parliament, Édimbourg : Polygon, 1999, p. 7.

15. La défense, les affaires étrangères, la monnaie, la gestion macro-économique, l'immigration et la nationalité, le commerce et l'énergie, l'emploi et le transport (Annexe 5).

16. Il ne peut modifier les articles 4 et 6 du traité d'Union relatifs à la liberté du commerce, les lois de 1972 et de 1998 qui transposaient respectivement les traités communautaires et la CEDH dans l'ordre juridique interne, les lois relatives aux domaines réservés et le Scotland Act 1998 luimême (Article 29). De plus, ses immunités ne sont pas générales et doivent être précisées (Articles 41 et 42 ).

17. Elle affecte notamment l'Écosse dans ses aspects sociaux (cf. J. McFADDEN \& M. LAZAROWICZ, The Scottish Parliament, Édimbourg : Clark, pp. 82-83) ; de même, les discussions britanniques sur l'abaissement de l'âge nubile dans les relations homosexuelles ont évité aux Écossais des débats difficiles (cf. P. JONES "The First Laws of the New Scottish Parliament" in G. HASSAN et al, op. cit., p. 64). Par ailleurs, certains domaines sont officiellement partagés : la santé est principalement gérée en Écosse, à l'exception de la réglementation des professions médicales, des médicaments et de la génétique, compétences britanniques (Annexe 5).

18. Il s'agit du ministre responsable et du Président du Parlement (lors du dépôt du projet), des conseillers juridiques écossais et du conseiller britannique ou Advocate General for Scotland, créé avec la réforme et enfin du Secrétaire d'État pour l'Écosse qui vérifie également, avant la promulgation, la conformité du texte à la politique internationale du Royaume-Uni (Articles 31, 32, 35). Ce dernier peut empêcher le Président du Parlement de soumettre le texte à l'assentiment royal, ce qui conduirait les conseillers juridiques à saisir le Judicial Committee of the 
Privy Council, juridiction constitutionnelle non pour la Grande-Bretagne (où le Parlement étant considéré comme souverain, ses actes ne sauraient être remis en cause en vertu d'un texte ayant valeur supérieure), mais pour certains pays du Commonwealth. En pratique, il sera composé uniquement des Lords juges britanniques (dont deux Écossais). Ses décisions s'imposent au Parlement écossais et à toute juridiction. De plus, le Judicial Committee of the Privy Council peut également être saisi, directement ou en appel, à l'issue de la promulgation d'un texte de loi, au cours d'un procès devant un tribunal ordinaire, soulevant un problème de compétence ou de légalité d'un acte, ou d'un défaut d'acte, de l'exécutif ou du législatif (Articles 34 et 103 et Annexe 6).

19. Mais le Judicial Committee of the Privy Council va prochainement devoir se prononcer sur une question de compatibilité avec la loi de 1998 sur les Droits de l'homme de la première loi votée en urgence par le Parlement écossais qui souhaitait, à l'initiative de l'exécutif, empêcher la sortie de prison d'autres meurtriers malades mentaux estimés incurables, suite à la victoire de Noel Ruddle en appel d'une décision du Court of Session. Plus haute juridiction civile d'Écosse, le Judicial Committee of the Privy Council est régulièrement saisi de ces questions, assimilées aux matières "dévolues ", car l'Écosse a donné force obligatoire à la Convention européenne plus tôt que le reste du Royaume-Uni (The Herald, 21/5/01, p. 4).

20. Par ailleurs, l'exécutif partage avec le gouvernement central la responsabilité de secteurs spécifiques (la formation et l'emploi). Il dispose néanmoins de pouvoirs supplémentaires s'agissant de certains domaines réservés. La fixation des limites de vitesse, par exemple, relève de sa compétence même si l'essentiel de la politique des transports lui échappe.

21. Le gouvernement central souhaitait conserver le monopole de ces désignations. H. McLeish souligne néanmoins que le First Minister, nommé par la reine, exerce des fonctions analogues à celles d'un Prime Minister.

22. Il s'agit de textes régissant leurs relations (protocole d'entente et concordats) et d'organes de coopération entre ministères (les plus formels étant les Joint Ministerial Committees). Leurs débats ne sont pas publiés.

23. Elle représente plus de la moitié de la pêche britannique. Les Écossais ont également assisté à des Conseils européens sur l'agriculture, l'environnement, l'éducation, soit huit au total la première année selon R. HAZELL “Intergovernmental Relations: Whitehall Rules, OK?" in R. HAZELL, op. cit., p. 173.

24. Ils ont adressé des rapports écrits (alors que Wendy Alexander, ministre écossaise des Affaires sociales, s'était rendue à Westminster), mais depuis le début de l'année 2001, leur déplacement à Édimbourg est encouragé (The Herald, 7/4/01, p. 2).

25. Les responsables procèdent à la répartition des crédits correspondant aux dépenses identifiables (par opposition aux dépenses non identifiables comme la défense) sur la base des besoins observés, notamment en ce qui concerne la sécurité sociale, qui représente le tiers des crédits de ce type, les autres dépenses étant couvertes par l'application de la formule Barnett, conçue en 1978 pour assurer un financement stable à l'Assemblée alors en projet. Voir The Constitution Unit, Scotland's Parliament, Londres : University College, 1996, chapitre 5.

26. Les crédits attribués au titre de cette formule sont fondés sur la part de l'Écosse dans la population britannique (10,66\%), et s'ajoutent à sa subvention globale, proche de 18 milliards de livres.

27. Cet objectif était lié à la diminution de la part des Écossais dans la population britannique et à la progression des dépenses publiques. Mais il n'a pas été atteint, d'une part, parce que l'affaiblissement du poids démographique de l'Écosse n'a pas été pris en compte avant les années quatre-vingt dix et, d'autre part, du fait de la quasi-stagnation des dépenses publiques en valeur réelle. Le gouvernement prévoit une progression annuelle moyenne de $4,4 \%$ en termes réels pour l'Écosse, contre $6 \%$ pour l'Angleterre (The Herald, 19/7/00, p. 1), et a réaffirmé, en juin 2001, qu'il s'engageait à assumer l'essentiel du financement des institutions écossaises. 
28. Cette limite tient à la nature de l'impôt (impôt sur le revenu), à la marge d'ajustement autorisée (+/- $3 \%$ ) et à son origine (décision du Parlement britannique). L'application du taux de majoration maximal ( $3 \%$ ) ne produirait d'ailleurs que 400 à 500 millions de livres de recettes supplémentaires, dont il faudrait déduire les frais de collecte.

29. Le recours à l'emprunt est limité aux cas de déséquilibre budgétaire temporaire (article 66), bien qu'il soit difficilement concevable que les collectivités locales, dont les ressources proviennent à plus de $80 \%$ d'Édimbourg, connaissent une réduction substantielle de leurs crédits.

30. Les comptes publics écossais sont vérifiés par un Auditeur général pour l'Écosse, et doivent faire l'objet d'une justification régulière tant en ce qui concerne le financement de projets en cours que l'utilisation effective des crédits (articles 69 et 70).

31. The Herald, $8 / 1 / 01$, p. 8 . L'augmentation, de $20 \%$, encouragera effectivement les candidats, parfois même non écossais.

32. Le nombre minimal de MP écossais (71), et l'utilisation d'un quotient électoral spécifique seront supprimés à partir du prochain découpage électoral (article 86) ; en outre, le lien entre les circonscriptions écossaises et celles servant aux élections législatives nationales (Annexe 1) pourrait bien ne pas être maintenu si l'on croit certains projets.

33. Cf. le journaliste de The Herald, M. RITCHIE, Scotland Reclaimed, Édimbourg: The Saltire Society, 2000, p. 212.

34. Seuls deux de ses collègues, S. Galbraith et D. Dewar, n'ont pu effectuer de choix. Entre 1999 et 2001, le taux de participation de ces MP-MSP aux votes à Londres était inférieur à $10 \%$, alors qu'il est proche de $50 \%$ en moyenne pour les MP écossais (The Herald, 7/3/01, p. 6). Voir aussi les observations recueillies par M. WATSON, op. cit., pp. 117-124.

35. La question porte le nom de la circonscription du député qui la soulève régulièrement depuis plus de vingt ans, Tam Dalyell, désormais doyen de Westminster. Seuls les conservateurs proposaient la suppression de ce double droit de vote en juin 2001, sans succès, tout comme le député travailliste Frank Field, auteur d'une proposition de loi sur le sujet.

36. On pense également à George Robertson (Défense, jusqu'à sa nomination à l'OTAN), ou Alistair Darling (Sécurité sociale).

37. Ils ont empêché la fermeture des chantiers navals de Govan (grâce aux contacts personnels du ministre britannique des Transports, Gus MacDonald), mais n'ont pu faire de même pour l'usine Motorala de Bathgate, malgré l'intervention de Tony Blair (The Herald, 11/4/01, p. 1 et 11/6/01, p. 6).

38. Voir l'opposition au projet d'édition écossaise du journal du soir de la BBC, ou la défense de décisions britanniques controversées in P. SCHLESINGER, D. MILLER, W. DINAN, Open Scotland?, Édimbourg : Polygon, 2001, pp. 92 et 174.

39. La question d'un regroupement du Scottish Office avec un autre ministère resurgit régulièrement à Westminster. Certains projets travaillistes envisagent sa fusion avec un futur ministère des affaires régionales. Pour leur part, les conservateurs proposaient en juin 2001 de le regrouper avec un ministère à caractère sectoriel.

40. Ce dernier se serait prévalu, au sein de son cabinet de relations avec des personnalités publiques, de son accès privilégié et de son influence auprès des institutions écossaises ; l'affaire a été surnommée "Lobbygate " par des médias devenus acteurs, car elle a été soulevée par The Observer, tandis que The Scotsman a fait pression au plan judiciaire pour obtenir la publicité des travaux de la commission concernée. P. SCHLESINGER et al., op.cit., p. 236.

41. Volontiers autoritaire, Tony Blair ne cherche pas à imposer ses vues s'agissant des affaires écossaises. Conscient des contraintes auxquelles sont confrontés les premiers ministres écossais, il en apprécie la modération et entretient d'étroites relations avec leurs principaux conseillers.

42. C'est le cas du ministre de l'Intérieur Jack Straw, qui a travaillé en étroite coopération avec Jim Wallace, ou du ministre de l'Agriculture Nick Brown avec Ross Finnie; ce dernier a même 
reçu des excuses de son homologue qui avait tardé à l'informer de la vente, par mégarde, de graines contenant des organismes génétiquement modifiés.

43. W. Alexander a entrepris rapidement des démarches de reclassement des salariés et de recouvrement d'une partie de l'aide publique reçue par Motorala pour son site de Bathgate (The Herald, 26/4/01, p. 1), mais a été plus discrète au sujet de l'intervention du gouvernement lors de la reprise du site de Huyndai par cette même entreprise (Voir C. MACLEOD, "Relations with Westminster" in G. HASSAN et al, op.cit., p. 121).

44. L'autorisation de séjour en Écosse délivrée à Mike Tyson par les autorités britanniques a été jugée inopportune par le Parlement écossais, compte tenu de la personnalité du boxeur.

45. Il s'agit des sheriff temporaires, dont la nomination était jugée contraire à la CEDH, de même que la législation sur les pouvoirs d'enquête de la police, également réformée.

46. Le premier est demeuré inchangé en Angleterre, la seconde n'a pas abouti, et la troisième est plus restrictive.

47. On peut citer la recherche de mécanismes d'aide financière aux étudiants anglais, le débat sur les personnes âgées dépendantes pendant la campagne électorale 2001, voire la création d'un ministère des Affaires rurales à la suite des élections.

48. Tony Blair et son homologue Paddy Ashdown ont perçu un modèle éventuel pour la GrandeBretagne: le premier appréciait la présence d'un leader incontesté, maitrisant les subtilités locales, et le second appréciait d'avoir un parti décentralisé.

49. Les travaillistes sont très réticents; c'est d'ailleurs leur parti qui a réintroduit le paiement des droits universitaires. La question fut renvoyée à une commission indépendante, présidée par Andrew Cubie, qui proposera leur suppression considérée comme indispensable à la survie de la coalition, même si les travaillistes obtiendront une réduction du montant des bourses et du seuil de reversement. L'exécutif s'est également engagé à augmenter les dépenses dans des domaines prioritaires, comme l'éducation.

50. Ils obtiennent un cinquième de la vingtaine de postes, dont deux ministères, avec seulement un septième des sièges.

51. Ainsi, D. Steel n'aura pas à intervenir pour le désigner formellement.

52. H. McLeish, ministre chargé de la question des droits d'inscription universitaire, a pour délégué Nicol Stephen, issu du parti libéral-démocrate. A l'inverse, ce dernier obtient, en raison de son implantation, le portefeuille des Affaires rurales, confié à R. Finnie, assisté du travailliste John Home Robertson. De même, H. McLeish confie le ministère de l'Éducation à son collègue (et adversaire lors de la succession de D. Dewar) J. McConnell assisté de N. Stephen.

53. D. Dewar, H. McLeish, S. Galbraith, et Malcolm Chisholm (second exécutif). Peter Peacock, Frank McAveety (premier exécutif) et Tom McCabe (second exécutif), ont géré de grandes collectivités locales. Ils sont tous travaillistes.

54. Huit ont moins de quarante ans: W. Alexander, J. McConnell, Sarah Boyack, S. Deacon (ministres), Jackie Baillie, A. MacKay (ministres délégués devenus ministres), Alistair Morrison et Tavish Scott (demeurés ministres délégués). Seul ce dernier est libéral-démocrate. Toutefois, la moyenne d'âge des MSP ne se distingue guère de celle des MP.

55. Ils soulignaient la présence de conseillers pour les libéraux-démocrates, P. SCHLESINGER et al, op.cit., pp. 173-185.

56. Cela concerne les droits d'inscription, la liberté d'information, le mode de recrutement des juges, les péages autoroutiers, les mesures en faveur des personnes âgées, des enseignants et des pêcheurs, ou les allocations des députés élus au scrutin de liste, exemples dûment rappelés pendant la campagne électorale de juin 2001.

57. Même le ministre de la Santé, S. Deacon, était peu favorable à cette mesure. The Herald, 30/1/01, p. 6 .

58. L'opposition à l'exécutif a des raisons diverses. Elle peut tenir à des facteurs locaux liés à la circonscription d'origine du député (c'est notamment le cas de Nora Radcliffe et de Mike Rumbles 
élus dans l'Aberdeenshire), à des positions de principe (lorsque, par exemple, le groupe libéraldémocrate a refusé d'écarter l'amendement de l'exécutif à la proposition de Tommy Sheridan relative aux modalités de remplacement des droits universitaires). Elle peut, enfin, s'expliquer par des raisons plus générales comme dans le cas de Keith Raffan qui s'était opposé à la désignation de Donald Dewar et à la nomination de D. Gorrie à la Commission Cubie. M. WATSON, op.cit., pp. 74 et 103.

59. Les députés ont voté, grâce à la voix prépondérante de David Steel, un système d'aide ponctuelle aux pêcheurs alors contraints à l'arrêt pour préserver les ressources, présenté par le SNP en complément au projet de « retrait durable » de l'exécutif, à la suite de quoi T. Scott (L-D), ministre délégué aux relations avec le Parlement, a démissionné. The Herald, 16/3/01, p. 6.

60. Ils ne se sont pas associés au SNP pour voter une motion de défiance à l'égard du ministre des Transports S. Boyack sur la question pourtant controversée de l'attribution au secteur privé de contrats de maintenance des routes nationales (The Herald, 7/3/01, p. 6). Ils n'ont pas davantage emboîté le pas au SNP lorsque celui-ci s'est opposé au projet de loi sur le logement qui vient d'être adopté deux ans après son dépôt à la suite d'un rapport minoritaire, alors que certains travaillistes, tel John McAllion, refusent l'extension de la politique de vente des logements sociaux à leurs locataires (The Scotsman, 14/6/01, p. 1).

61. Le financement des institutions écossaises est devenu un enjeu, et W. Alexander s'occupait de la campagne en Écosse, en liaison avec son frère Douglas à Londres. De plus, l'électorat perçoit bien le rôle respectif des deux Parlements, même si celui de Westminster paraît moindre, sur des questions jugées essentielles, comme la santé et l'éducation (The Scotsman, 15/5/01, p. 1).

62. Lors de deux des élections partielles survenues depuis 1997, les libéraux-démocrates ont été devancés par le SNP, et l'avantage des travaillistes s'est volatilisé, au profit du SNP. Mais les travaillistes ont conservé leurs sièges lors des élections de juin 2001, tandis que le parti libéraldémocrate progressait en voix. De plus, le coefficient de corrélation entre leurs résultats dans les circonscriptions $(-0,75)$ indique l'absence de concurrence directe entre eux, ce qui a facilité leur travail au sein du Parlement écossais alors en session.

63. Ainsi, ils ont perdu un siège en 2001. L'efficacité de leur opposition semble compromise par leur refus de s'allier aux conservateurs, mais se trouve alimentée par l'absence de corrélation significative avec les résultats de tous leurs adversaires.

64. En effet, ils ont gagné un seul siège en juin 2001, et ce léger progrès par rapport à 1997 semble lié à leur récente défense des intérêts écossais, même si ceux-ci impliquent une augmentation des dépenses publiques.

65. Voir E. BORT “The Devolution Journey Continues”, Scottish Affairs, $\mathrm{n}^{\circ}$ 34, hiver 2001, p. 14, dont le titre reprend une phrase prononcée par Donald Dewar lors de l'inauguration du Parlement, qui contraste avec le pessimisme nourri par l'attitude de Tony Blair, et de T. NAIRN, After Britain, Londres : Granta Books, 2000, p. 82.

\section{RÉSUMÉS}

Le nouveau Parlement écossais élu en mai 1999 est fondé sur le partage du pouvoir, avec des commissions prééminentes qui contrôlent l'exécutif et qui sont ouvertes sur la société civile. Ainsi, les Écossais élaborent leurs propres orientations, dans le respect des limites et des adaptations requises par le processus de dévolution du pouvoir. 
The new Scottish Parliament elected in May 1999 is based on power-sharing, as it has powerful committees which hold the executive to account and respond to outside interests. The Scots have thus been working out their own policies, while abiding by the limits and the adjustments required by the devolution process.

\section{AUTEUR}

\section{EDWIGE CAMP}

Université de Valenciennes 